\title{
Epidemiology of viral hepatitis in men who have sex with men in Mediterranean Europe: a region-wide sero-surveillance in Rome (Italy)
}

\section{CURRENT STATUS: UNDER REVISION}

BMC Infectious Diseases $\triangle$ BMC Series

Simone Lanini

च simone.Ianini@inmi.itCorresponding Author

ORCiD: https://orcid.org/0000-0002-6743-4001

Silvia Meschi

INMI Lazzaro Spallanzani IRCCS

Laura Scorzolini

INMI Lazzaro Spallanzani IRCCS

Giovanna Adamo

INMI Lazzaro Spallanzani IRCCS

Alessandro Agresta

INMI Lazzaro Spallanzani IRCCS

Fabrizio Marrazzo

Gay Center/ Gay Help Line - Rome

Francesco Angeli

Arcigay - Rome

Massimo Farinella

Circolo di cultura omosessuale "Mario Mieli" - Rome

Carlo Guarino

Regione Lazio

Alessandra Barca

Regione Lazio

Lorella Lombardozzi

Regione Lazio 
Adriano Possi

INMI Lazzaro Spallanzani IRCCS

Mirko Manganiello

LAZIOcrea - Rome

Patrizia Magrini

INMI Lazzaro Spallanzani IRCCS

Paola Scognamiglio

INMI Lazzaro Spallanzani IRCCS

Vincenzo Puro

INMI Lazzaro Spallanzani IRCCS

Emanuele Nicastri

INMI Lazzaro Spallanzani IRCCS

Maria Capobianchi

INMI Lazzaro Spallanzani IRCCS

Alimuddin Zumla

University College London Division of Infection and Immunity

Giuseppe Ippolito

INMI Lazzaro Spallanzani IRCCS

\section{DOI:}

10.21203/rs.2.22953/v1

\section{SUBJECT AREAS}

Infectious Diseases

\section{KEYWORDS}

Hepatitis, Viral, Human, HAV, HBV, HCV, Men Who Have Sex With Men, Serosurveillance 
Abstract

OBJECTIVE To describe epidemiology of viral hepatitis (including HAV, HBV and HCV) among men who have sex with men (MSM) aged between 18 and 45 years and living in Lazio, an Italian Region with about 5.9 million inhabitants whose main city is Rome.

METHODS A sero-survey (cross-sectional study) was conducted as part of a screening and prevention campaign for improving control of viral hepatitis in Lazio. The study enrolled 1,100 MSM people participating to the campaign. Outcome measures were serology for HAV (anti-HAV IgG and anti-HAV IgM), serology for HBV (anti-HBs, anti-HBC and HBsAg), and serology for HCV (anti-HCV). Participants' epidemiological features were assessed for potential association with outcomes.

RESULTS We found a very low prevalence of immunity against HAV and a significant exposure to highrisk behaviours, suggesting that gay community of Lazio is an ideal setting for HAV outbreaks. Prevalence of different serology profiles for HBV suggests that waning immunity and escape to mandatory vaccination still represent a significant issue in patients older than 35 years. Moreover, waning immunity suggests that patients who were vaccinated at birth may show low antibody levels. Consistently with other studies, prevalence of HCV infection was very low among young MSM. CONCLUSION Tailored vaccine programme aimed at immunizing MSM are critical to improve control of HAV in our setting. Findings on HBV epidemiology suggest that there is a need to define a clear strategy to recover patients who escaped vaccination and inform clinicians on the need to booster subjects with low immunity and high risk of exposure, such as MSM. Finally, MSM in Lazio do not represent a special group for HCV neither in terms of risk of infection nor in terms of potential issues for therapy access.

\section{Full Text}

Due to technical limitations, full-text HTML conversion of this manuscript could not be completed. However, the manuscript can be downloaded and accessed as a PDF. Figures 


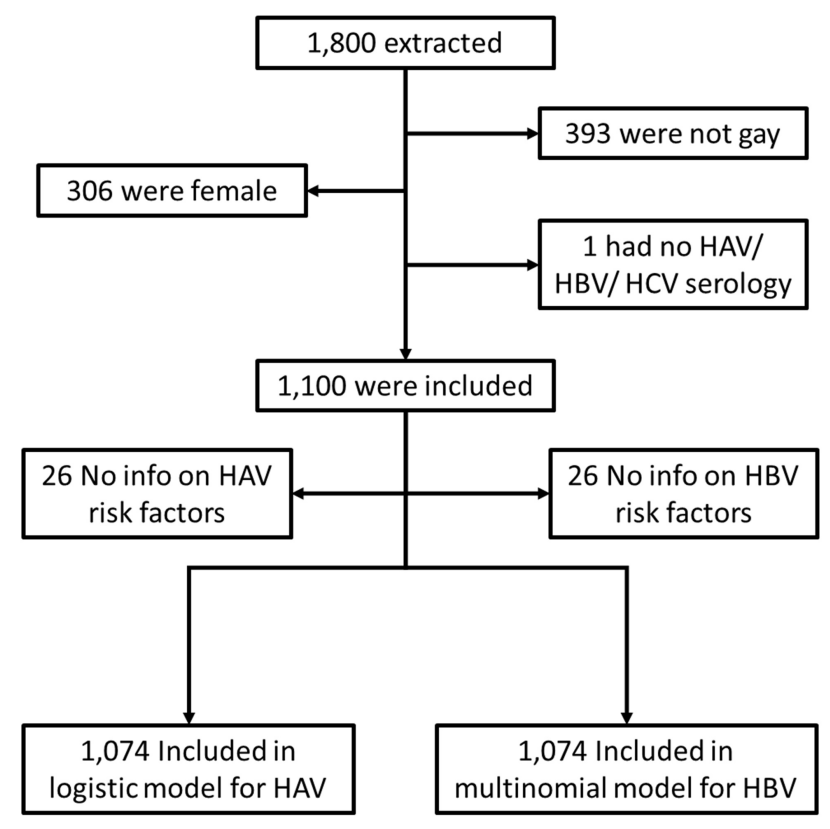

Figure 1

Flow chart for sample selection. 


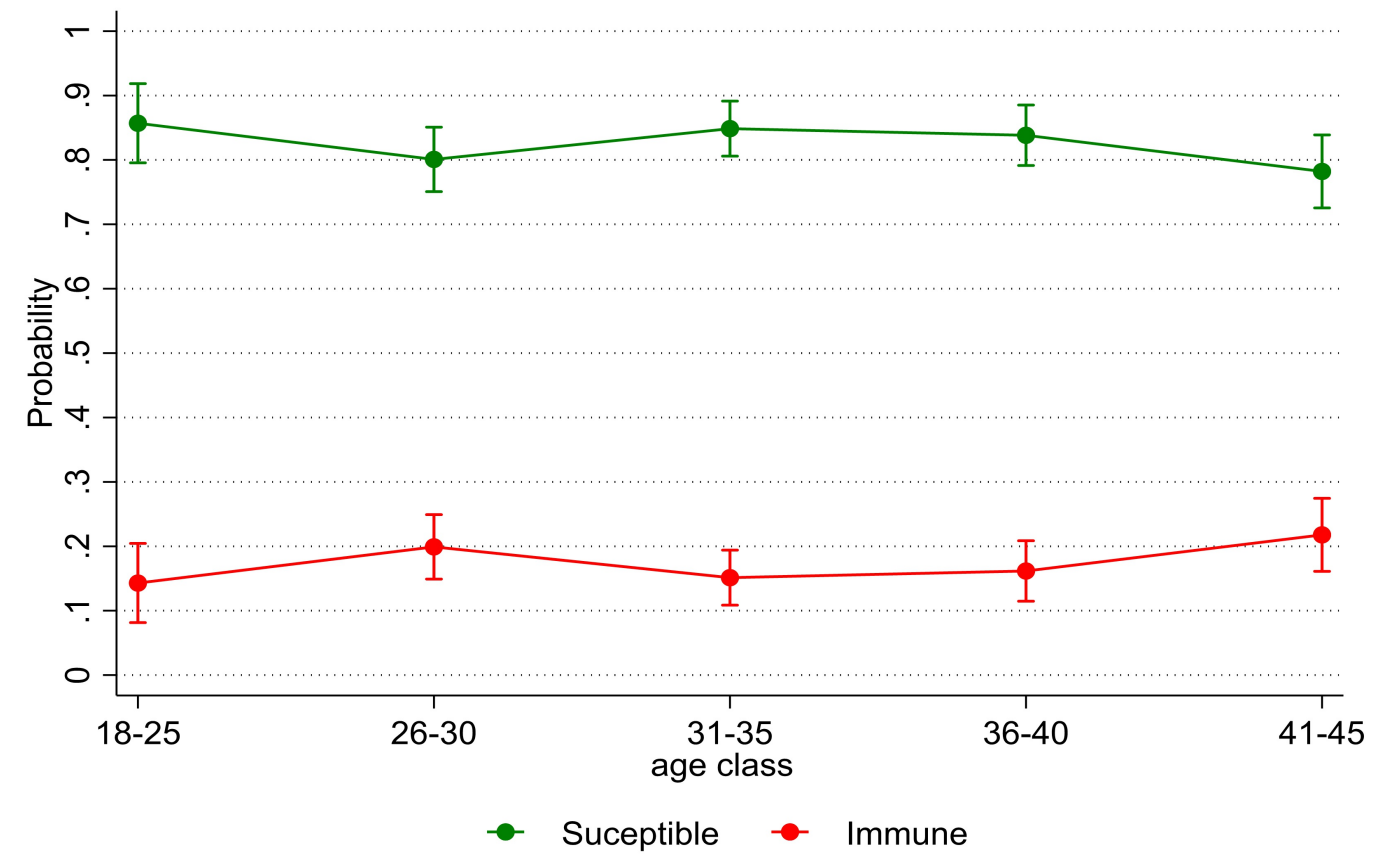

Figure 2

Estimated effect of age on the probability to show a specific HAV sero-status. Immune: detectable anti-HAV IgG and/or anti-HAV IgM. Susceptible: undetectable anti-HAV IgG and anti-HAV IgM antibodies. 


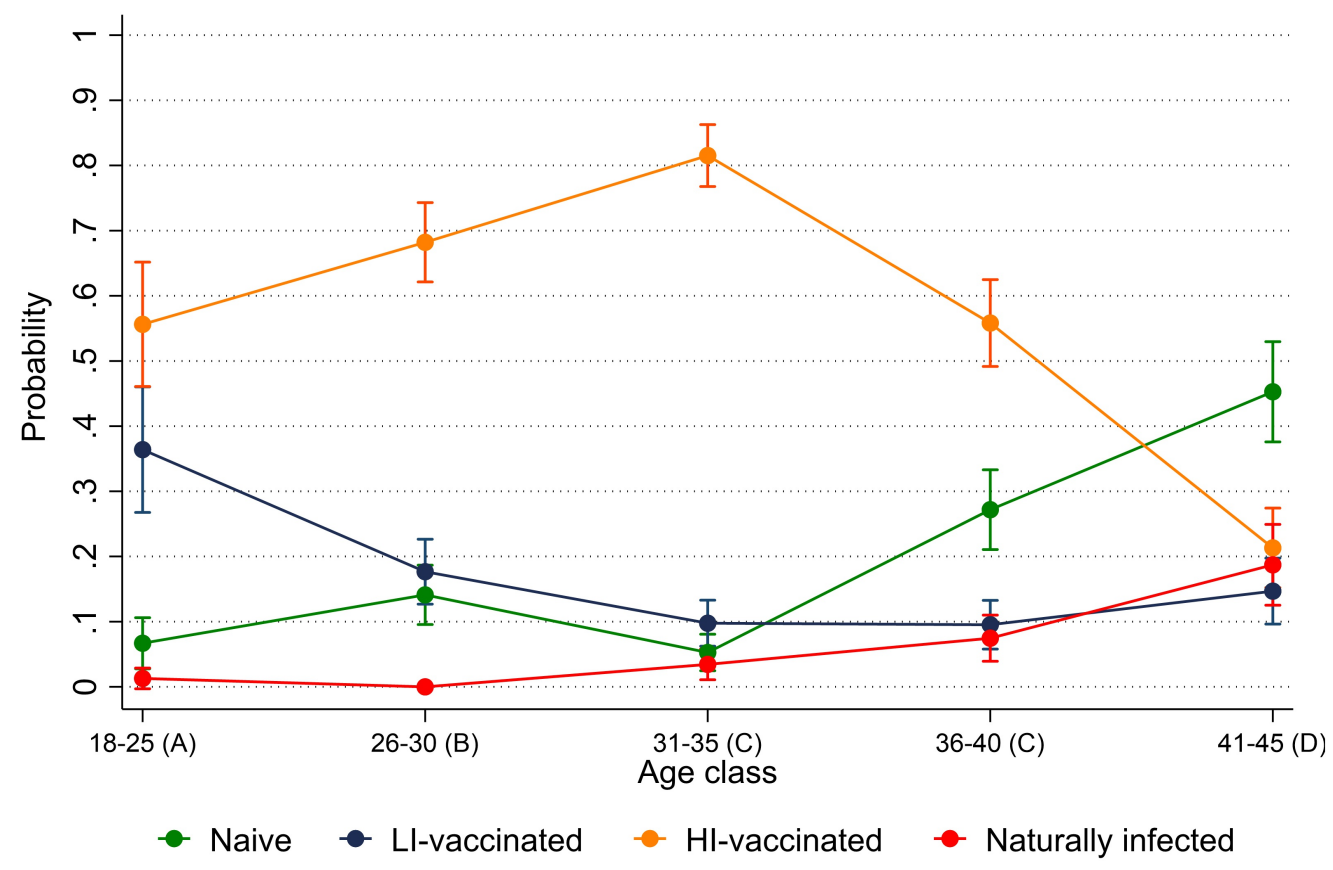

Figure 3

Estimated effect of age on the probability to show a specific HBV sero-status. (A) Age cohorts who received vaccination as infants. (B) Age cohorts who received vaccination either as infants or as 12-year children. (C) Age cohorts who received vaccination as 12-year children. (D) People born before implementation of universal vaccine programme. HBV naturally infected: detectable anti-HBcAb. HI-vaccinated: anti-HBs $>10 \mathrm{UI} / \mathrm{mL}$ and undetectable anti-HBc. LI-vaccinated: anti-HBs between 1 and $10 \mathrm{UI} / \mathrm{mL}$ and undetectable anti-HBc. Naïve: undetectable anti-HBs-Ab and anti-HBc.

\section{Supplementary Files}

This is a list of supplementary files associated with this preprint. Click to download.

Suppl_file_1.pptx

Suppl_file_3.docx

Suppl_file_4.docx

Suppl_file_2.docx 